\title{
HIV-1: nature's master of disguise
}

\author{
HIV-1 readily mutates to escape the immune response, evolving in ways that allow it to persist in the host. New \\ findings reveal that HIV-1 protects itself from antibodies by putting up a shield of constantly shifting sugar \\ moieties. This shield may be contributing to the poor performance of candidate HIV-1 vaccines.
}

$\mathrm{H}^{\mathrm{s}}$ IV-1 mutates to avoid recognition by the host immune response. This allows the virus to chronically replicate and to eventually wear down the body's defenses by destroying the very cells necessary to coordinate an effective immune response ${ }^{1}$. A key feature of immune escape is the ability of HIV-1 to conceal itself from neutralizing antibodies that would otherwise bind the virus and block its entry into cells. This stealth-like property may explain why antibodies generated by candidate HIV1 vaccines neutralize so few strains of the virus. This has frustrated attempts to develop a vaccine because neutralizing antibodies are believed to be a key component of the protective immune response. The disappointing results of the first phase 3 trial of a candidate AIDS vaccine, AIDSVAX (see page 376 ), based on the gp120 viral envelope glycoprotein, highlight how tough it is to generate a protective antibody response against HIV-1.

In the March 202003 issue of Nature, Wei et al. add a new level to our under-

\section{JOHN R. MASCOLA ${ }^{1} \&$ \\ DAVID C. MONTEFIORI ${ }^{2}$}

standing of the mechanisms used by HIV-1 to resist neutralization by circulating antibodies ${ }^{2}$. Specific genetic changes in the HIV-1 envelope glycoproteins seem to result in the acquisition and rearrangement of sugar moieties (asparagine-linked glycans), forging them into an evolving protective shield that prevents antibody binding.

Neutralization epitopes reside on the surface gp120 and transmembrane gp41 envelope glycoproteins, which are derived from a gp160 precursor molecule. These glycoproteins form a complex that mediates receptor and co-receptor binding and the subsequent membrane fusion events that permit virus entry ${ }^{3}$. HIV-1 is neutralized when antibodies bind critical regions on either glycoprotein, thereby disrupting the entry process.

During the course of infection, the virus survives by mutating to escape antibody recognition ${ }^{4}$. The viral escape variants elicit subsequent neutralizing antibody responses. Multiple rounds of neutralization-escape are thought to explain, in part, how HIV-1 persistently replicates in the host. A similar phenomenon occurs during infection with several other animal lentiviruses ${ }^{5-7}$.

HIV-1 neutralization escape is not always a simple matter of changing the contact amino acids in the cognate epitope. Some epitopes, such as those that directly bind the receptor, need to be preserved to maintain viral fitness. HIV 1 has devised several clever strategies to mask these conserved epitopes ${ }^{8}$. The receptor binding domains are positioned in recessed pockets on the gp120 molecule, where access to antibody is limited. Other epitopes are shielded by conformationally flexible tertiary folds on the gp120 molecule.

In addition, there are the asparagine (N)-linked glycans. HIV-1 gp120 contains approximately 25 sites for $\mathrm{N}$ linked glycosylation. These glycans are a

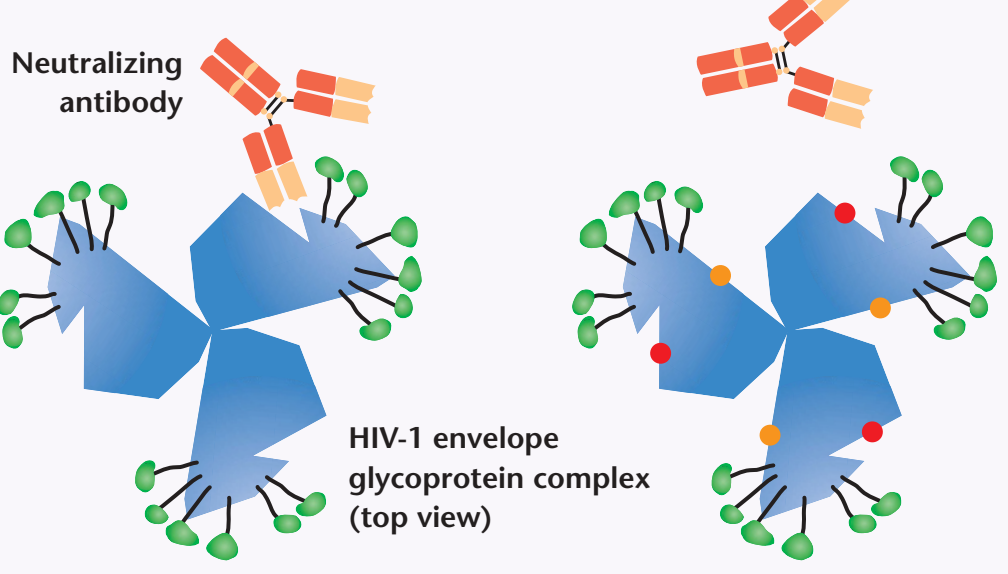

b c

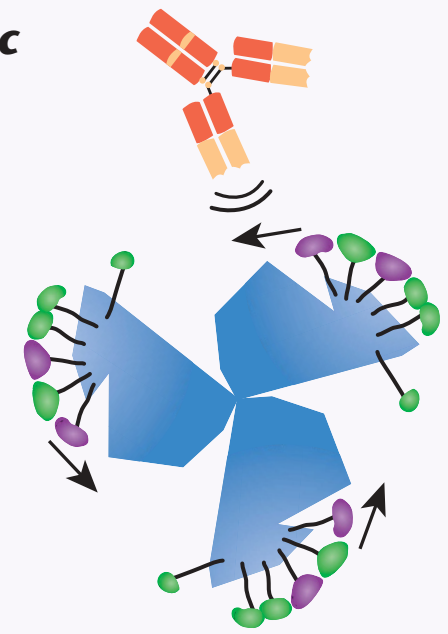

Fig. 1 A comparison of the direct epitope variation and the glycan shield models of HIV-1 immune escape. $\boldsymbol{a}$, Neutralizing antibodies bind to the viral envelope glycoprotein complex, which consists of 3 identical gp120-gp41 heterodimers. b. Traditional viral escape involves random mutations (resulting envelope changes depicted with red and orange dots) that alter the neutralizing epitope (red dots) and diminish antibody binding. $\boldsymbol{c}$, The glycan shield model proposed by Wei et al. suggests that steric hindrance rather than epitope variability prevents neutralizing antibodies from binding their cognate epitopes. Added or repositioned glycans are shown in purple. The gp120 glycoprotein can accommodate only a limited number of carbohydrates, so the shifting of glycans to occlude one epitope seems to leave other regions vulnerable. Moreover, complete shielding would interfere with critical virus-cell interactions and result in a defective virus. Thus, neutralization escape mutants adapt to neutralizing antibodies but do not become completely neutralization resistant. Ultimately, viral immune escape probably results from a combination of mechanisms that balance viral fitness with evasion from both humoral and cellular immune responses. 
complex branched-chain carbohydrate structures that in total account for approximately $50 \%$ of the molecular mass of the glycoprotein. The removal of specific glycan sites on the HIV-1 glycoprotein can dramatically increase viral sensitivity to neutralizing antibodies ${ }^{9}$.

Wei et al. explored the nature of the changes in the envelope glycoproteins by sequencing gp160 genes from multiple escape variants that arose after primary HIV-1 infection. These changes were correlated to the neutralization phenotype of the virus.

Their data provide the first systematic correlation of env gene sequence changes in multiple neutralizationescape variants that arise during the course of HIV-1 infection in humans. Their study was facilitated by the use of a pseudotype virus neutralization assay that can readily produce viruses containing gp160 encoded by sequential plasma-derived env genes.

The investigators identified gp160 mutations in sequential escape variants from an individual designated WEAU. Strikingly, a high frequency of changes occurred in sites that determine $\mathrm{N}$ linked glycan addition. Some changes fixed the position of N-linked glycans over time, as if to preserve the architecture needed for escape from earlier antibodies, while other sites were either introduced or repositioned as additional rounds of escape were detected. Sequence analysis of an escape variant from a second study subject again showed multiple changes in the pattern of N-linked glycosylation.

Because gp120 can accommodate only a limited number of sugar moieties, strategic positioning involves a combination of additions, removals and minor shifts in sites-hence the authors' reference to an "evolving glycan shield" that sterically hinders antibody binding but does not reduce viral fitness (Fig. 1).

Further support for this model came from site-directed mutagenesis studies. The investigators found that early viruses could acquire a neutralizationescape phenotype by introducing the glycosylation pattern of the corresponding escape variant. To achieve a full phenotypic switch, mutations in gp120 that did not alter glycosylation were also sometimes required.

The authors suggest that such nonglycan mutations could contribute to alterations in the overall "packing" of the glycan shield, but it is also possible that they affect the conformational antigenic structure of envelope glycoprotein, thus reducing antibody recognition.

Three other groups have recently reported the rapid emergence of escape variants in HIV-1 infected individuals; two also found that sequential shifts in $\mathrm{N}$-linked glycan sites corresponded to the development of neutralization escape (refs. 10, 11 and K.J. Weinhold, M. Greenberg, G. Tomaras, personal communication).

The glycan shield hypothesis is an attractive model because it attempts to explain several confounding observations. For example, viral escape mutants resist neutralization by a polyclonal antibody response in autologous plasma, but such viruses are not globally neutralization resistant. In fact, they remain sensitive to certain gp160-specific monoclonal antibodies and to plasma from other HIV-1 infected patients ${ }^{4,12}$. Similarly, plasma antibodies that are completely ineffective against the autologous virus can often neutralize heterologous viruses.

Thus, the evolving glycan shield model offers an explanation of how the virus can effectively adapt to evade the neutralizing antibody specificities of a particular host without developing a global neutralization-resistant phenotype. In other words, the virus is shielded from antibodies, but the shield has holes.

This hypothesis may provide only one part of the story of HIV-1 neutralization escape. Amino acid variation can directly alter antibody binding and epitopes may be further concealed by the tertiary and quaternary structures of the trimeric envelope complex. Ultimately, viral immune escape probably results from a combination of mechanisms that balance viral fitness with evasion from both humoral and cellular immune responses.

To date, HIV-1 vaccines based on components of envelope glycoproteins have not generated potent neutralizing antibodies. Mutations that evolve under immune selection pressure in infected individuals probably shield the virus from the antibodies generated by current vaccine candidates, limiting the ability of these antibodies to prevent transmission or protect against HIV-1 disease. Thus, a detailed understanding of the mechanisms used by HIV-1 to resist antibody-mediated neutralization could have major implications for vaccine development. Investigators are attempting to use this knowledge, along with the atomic structure of gp160, to engineer vaccine constructs that could more effectively present key neutralization epitopes to the immune system.

HIV-1 neutralization escape has taught us that the virus has a daunting arsenal of mechanisms for evading antibodies. Nevertheless, the virus is vulnerable and certain antibodies can neutralize HIV-1. A more detailed understanding of viral escape mechanisms, as provided here by Wei et al., may bring us one step closer to the rational design of an effective vaccine.

1. Letvin, N.L., Barouch, D.H. \& Montefiori, D.C. Prospects for vaccine protection against HIV-1 infection and AIDS. Annu. Rev. Immunol. 20, 73-99 (2002)

2. Wei, X. et al. Antibody neutralization and escape by HIV-1. Nature 422, 307-312 (2003).

3. Wyatt, R. \& Sodroski, J. The HIV-1 envelope glycoproteins: fusogens, antigens, and immunogens. Science 280, 1884-1888 (1998).

4. Albert, 1. et al. Rapid development of isolate-specific neutralizing antibodies after primary HIV-1 infection and consequent emergence of virus variants which resist neutralization by autologous sera. AIDS 4, 107-112 (1990).

5. Narayan, O., Griffin, D.E. \& Chase, J. Antigenic shift of visna virus in persistently infected sheep. Science 197, 376-378 (1977).

6. Howe, L., Leroux, C., Issel, C.J. \& Montelaro, R.C. Equine infectious anemia virus envelope evolution in vivo during persistent infection progressively in creases resistance to in vitro serum antibody neutralization as a dominant phenotype. J. Virol. 76 10588-10597 (2002).

7. Burns, D.P. Collignon, C \& Desrosiers, R.C. Simian immunodeficiency virus mutants resistant to serum neutralization arise during persistent infection of rhesus monkeys. J. Virol. 67, 4104-4113 (1993).

8. Kwong, P.D. et al. HIV-1 evades antibody-mediated neutralization through conformational masking of receptor-binding sites. Nature 420 678-682 (2002).

9. Kolchinsky, P., Kiprilov, E. \& Sodroski, J. Increased neutralization sensitivity of CD4-independent human immunodeficiency virus variants. I Virol. 75, 2041-2050 (2001).

10. Montefiori, D.C. et al. Viremia control despite escape from a rapid and potent autologous neutralizing antibody response after therapy cessation in an HIV-1 infected individual. J. Immunol. (in the press).

11. Richman, D.D., Wrin, T.L., Little, S.J. \& Petropoulos, C.J. Rapid evolution of the neutralizing antibody response to HIV-1 infection. Proc. Natl. Acad. Sci. USA (in the press)

12. Wrin, T. et al. Neutralizing antibody responses to autologous and heterologous isolates of human immunodeficiency virus. J. Acquir. Immune. Defic Syndr. 7, 211-219 (1994)

${ }^{1}$ Vaccine Research Center, National Institute of Allergy and Infectious Diseases, National

Institutes of Health, Bethesda, Maryland, USA ${ }^{2}$ Department of Surgery,

Duke University Medical Center,

Durham, North Carolina

Email:jmascola@mail.nih.gov 